University of Warwick institutional repository: http://go.warwick.ac.uk/wrap This paper is made available online in accordance with publisher policies. Please scroll down to view the document itself. Please refer to the repository record for this item and our policy information available from the repository home page for further information.

To see the final version of this paper please visit the publisher's website. Access to the published version may require a subscription.

Author(s): MARK POLLICOTT and RICHARD SHARP

Article Title: Addendum: An analogue of Artin reciprocity for closed orbits of skew products

Year of publication: 2008

Link to published version: http://dx.doi.org/

10.1017/S0143385707000569

Publisher statement: None 


\title{
Addendum: An analogue of Artin reciprocity for closed orbits of skew products
}

\author{
MARK POLLICOTT $\dagger$ and RICHARD SHARP $\ddagger$ \\ $\dagger$ Department of Mathematics, University of Warwick, Coventry CV4 7AL, UK \\ (e-mail: m.pollicott@warwick.ac.uk) \\ \$ School of Mathematics, University of Manchester, Oxford Road, Manchester, \\ M13 9PL, UK \\ (e-mail:sharp@maths.man.ac.uk)
}

(Received 26 April 2007 and accepted in revised form 24 July 2007)

William Parry FRS, in memoriam

Abstract. One of the unfulfilled aims of the authors of the preceding paper [W. Parry and M. Pollicott. An analogue of Bauer's theorem for closed orbits of skew products. Ergod. Th. \& Dynam. Sys. 28 (2008), 535-546] was to find a dynamical analogue of Artin reciprocity. In this addendum, we present one such version, suggested by work of Sunada.

\section{Introduction}

In algebraic number theory, one of the most important themes is that of reciprocity. Historically, this originated with the famous quadratic reciprocity theorem, conjectured by Euler and proved by Gauss. This relates the existence of solutions to quadratic equations $x^{2}=p(\bmod q)$ to the existence of solutions to quadratic equations $x^{2}=q$ $(\bmod p)$. Various extensions and generalizations culminated in Artin's reciprocity theorem, proved in 1927 [2], from which quadratic reciprocity can be deduced (albeit after some effort). This is a key result in class field theory.

Let $K$ be the base field and let $L=K(\alpha)$ be a finite extension with Galois group $\operatorname{Gal}(L / K)$. The Artin reciprocity theorem describes the Galois group $\operatorname{Gal}(L / K)$ in terms of properties of the base field $K$. More precisely, it gives an isomorphism

$$
\mathcal{I} / \mathcal{P N} \cong \operatorname{Gal}(L / K) \text {. }
$$

Here $\mathcal{I}$ are fractional ideals coprime to an admissible cycle $\mathcal{C}$ in $L ; \mathcal{P}$ are the associated principal fractional ideals; and $\mathcal{N}$ is the group of fractional ideals of the form $N(\mathcal{B})$, where $\mathcal{B}$ is a fractional ideal which is prime to $\mathcal{C}$ (and $N$ denotes the norm). We refer the reader to [1] for details.

In this addendum to [4] we present analogous results for subshifts of finite type and hyperbolic flows where closed orbits play the role of prime ideals. In this context, the use of homology leads to simplifications not available in the original number theoretical context. 


\section{Skew products of subshifts}

Let $\sigma: \Sigma \rightarrow \Sigma$ be a mixing subshift of finite type, based on the alphabet $\{0, \ldots, k-1\}$. We first want to introduce a group which is analogous to the fractional ideal group. In particular, we associate to the shift $\sigma: \Sigma \rightarrow \Sigma$ a free abelian group $\mathcal{I}(\sigma)$ generated by closed orbits, i.e. a typical element is of the form $\tau_{1}^{n_{1}} \cdots \tau_{k}^{n_{k}}$, where $\tau_{1}, \ldots, \tau_{k}$ are closed orbits and $n_{1}, \ldots, n_{k} \in \mathbb{Z}$.

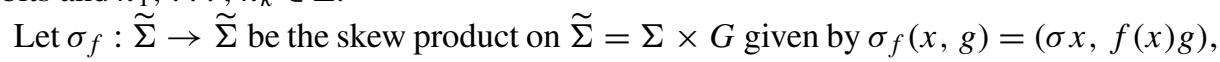
where $G$ is a finite group and $f: \Sigma \rightarrow G$ is a continuous map. We can assume without loss of generality that $f(x)=f\left(x_{0}, x_{1}\right)$, i.e. $f$ depends on the first two coordinates in the sequence. Clearly, $\widetilde{\Sigma}$ can also be viewed as a subshift of finite type, with alphabet $\{0, \ldots, k-1\} \times G$, and we shall also assume that $\sigma_{f}$ is mixing, i.e. the extension is Galois. This is equivalent to ergodicity of $\sigma_{f}$.

We can associate to each closed orbit $\tau=\left\{x, \sigma x, \ldots, \sigma^{n-1} x\right\}$ in $\Sigma$ a conjugacy class $[\tau]_{G}$ containing $f^{n}(x)=f\left(\sigma^{n-1} x\right) \cdots f(\sigma x) f(x)$, called the Frobenius class.

It is now convenient to take a slightly more geometric viewpoint. Let $\mathcal{G}^{+}$be the directed graph associated to $\Sigma$, i.e. $\mathcal{G}^{+}$has vertex set $\{0, \ldots, k-1\}$ and there is a directed edge from $i$ to $j$ if and only if $i j$ is an allowed word. By removing the arrows on the edges we obtain an undirected graph $\mathcal{G}$. In particular, if $\mathcal{G}^{+}$has a directed edge from $i$ to $j$ and a directed edge from $j$ to $i$ then $\mathcal{G}$ has two (undirected) edges joining $i$ and $j$. We can regard $\mathcal{G}$ as a 1 -complex.

Similarly, we can associate to $\widetilde{\Sigma}$ a directed graph $\widetilde{\mathcal{G}}^{+}$and an undirected graph $\widetilde{\mathcal{G}}$. Recall that there is a directed edge in $\widetilde{\mathcal{G}}^{+}$from $(i, h)$ to $\left(j, h^{\prime}\right)$ if and only if there is a directed edge in $\mathcal{G}^{+}$from $i$ to $j$ and $h^{\prime}=f(i, j) h$.

The following may be viewed as a geometric analogue of Proposition 4.1 of [4].

LEMMA 1. There is a natural one-to-one correspondence between mixing finite group extensions of $\sigma$ (recoded so that the skewing function depends on only two coordinates) and finite Galois (or regular) covers of $\mathcal{G}$.

Proof. Given a finite group $G$, let $\sigma_{f}: \widetilde{\Sigma} \rightarrow \widetilde{\Sigma}$ be a mixing $G$-extension of $\sigma: \Sigma \rightarrow \Sigma$ and, as above, let $\widetilde{\mathcal{G}}$ denote the undirected graph associated to $\widetilde{\Sigma}$. We claim that $\widetilde{\mathcal{G}}$ is a Galois $G$-cover of $\mathcal{G}$. Recalling that the vertex set of $\widetilde{\mathcal{G}}$ is $\{0, \ldots, k-1\} \times G$, we define a $G$-action on $\widetilde{\mathcal{G}}$ by $g(i, h)=(i, h g)$, for $g \in G$. Suppose that there is a directed edge in $\widetilde{\mathcal{G}}^{+}$from $(i, h)$ to $\left(j, h^{\prime}\right)$. Then $h^{\prime}=f(i, j) h$ or, equivalently, $h^{\prime} g=f(i, j) h g$, for any $g \in G$. Hence there is a directed edge from $(i, h g)$ to $\left(j, h^{\prime} g\right)$ and so the action on $\widetilde{\mathcal{G}}$ is well defined. It is then clear that $\widetilde{\mathcal{G}} / G=\mathcal{G}$.

Conversely, suppose that $\widetilde{\mathcal{G}}$ is a Galois $G$-cover of $\mathcal{G}$. Up to a choice of a fundamental domain, we may identify the vertices of $\widetilde{\mathcal{G}}$ with $\{0, \ldots, k-1\} \times G$ in such a way that $G$ acts by $g(i, h)=(i, h g)$. We define a mixing $G$-extension of $\sigma: \Sigma \rightarrow \Sigma$ as follows. Define $f: \Sigma \rightarrow G$ by $f(i, j)=g$ if $i j$ is an allowed word and if there is an edge in $\tilde{\mathcal{G}}$ joining $(i, 1)$ and $(j, g)$ (or, equivalently, an edge joining $(i, h)$ and $(j, g h)$ for any $h \in G)$. To see that $\sigma_{f}$ is mixing, it suffices to show that, given $(i, 1),(j, g) \in\{0, \ldots, k-1\} \times G$, we can find an allowed word $\left(x_{0}, 1\right),\left(x_{1}, g_{1}\right), \ldots,\left(x_{n-1}, g_{n-1}\right),\left(x_{n}, g\right)$, where $x_{0}=i$ and $x_{n}=j$. Since $\widetilde{\mathcal{G}}$ is connected, there is an undirected path joining $(i, 1)$ to $(j, g)$. However, some of its edges may be oriented 'the wrong way' so they do not correspond 
to a directed edge in $\widetilde{\mathcal{G}}^{+}$. Let us assume that $\left((x, h),\left(x^{\prime}, h^{\prime}\right)\right)$ is one such edge; then, in particular $\left(x^{\prime}, x\right)$ is an edge in $\mathcal{G}^{+}$. Since $\sigma$ is mixing, we can find a path in $\mathcal{G}^{+}$ from $x$ to $x^{\prime}$, which, when concatenated with $\left(x^{\prime}, x\right)$, gives a closed path $\tau\left(x, x^{\prime}\right)$. Now this will lift to a closed path in $\widetilde{\mathcal{G}}^{+}$(starting and ending at $(x, h)$ ) if and only if the Frobenius class $\left[\tau\left(x, x^{\prime}\right)\right]_{G}=1$. More generally, we iterate $\tau\left(x, x^{\prime}\right)|G|$ times, to obtain a closed path $\tau\left(x, x^{\prime}\right)^{|G|}$ with trivial Frobenius class and hence a closed path $\tilde{\tau}$ in $\widetilde{\mathcal{G}}^{+}$. Furthermore, by construction, the last edge in $\tilde{\tau}$ joins $\left(x^{\prime}, h^{\prime}\right)$ to $(x, h)$. Finally, we replace the (incorrectly oriented) edge $\left(x, x^{\prime}\right)$ by the other edges in $\tilde{\tau}$, to give a path in $\widetilde{\mathcal{G}}^{+}$ from $(x, h)$ to $\left(x^{\prime}, h^{\prime}\right)$, as required.

By analogy with Artin's theorem, we shall now specialize to abelian covers. When $G$ is abelian, $[\tau]_{G}$ is a well-defined element of $G$ and this correspondence extends in a natural way to a homomorphism $p_{G}: \mathcal{I}(\sigma) \rightarrow G$.

Proposition 1. The homomorphism $p_{G}: \mathcal{I}(\sigma) \rightarrow G$ is surjective.

Proof. It suffices to show that for any $g \in G$ we can find a closed orbit $\tau$ for $\sigma$ such that $p_{G}(\tau)=g$. Since, by hypothesis, $\sigma_{f}$ is mixing, we can find an admissible word $\left(x_{0}, 1\right),\left(x_{1}, g_{1}\right), \ldots,\left(x_{n-1}, g_{n-1}\right),\left(x_{0}, g\right)$ in the alphabet $\{0, \ldots, k-1\} \times G$. In particular, we can then associate to the word $x_{0} x_{1}, \ldots, x_{n-1}$ a closed orbit $\tau$ for $\sigma$ whose Frobenius element is precisely $g$.

By Lemma 1, the finite abelian Galois coverings of $\mathcal{G}$ then correspond precisely to finite quotients of the homology group $H_{1}(\mathcal{G}, \mathbb{Z})$. Let $p: \mathcal{I}(\sigma) \rightarrow H_{1}(\mathcal{G}, \mathbb{Z})$ be the homomorphism that comes from extending the map which associates to each closed orbit $\tau$ the homology class of the corresponding cycle in $\mathcal{G}$. The following follows from Proposition 1.

COROLlary 1. The homomorphism $p: \mathcal{I}(\sigma) \rightarrow H_{1}(\mathcal{G}, \mathbb{Z})$ is surjective.

Proof. The following argument is similar to one used in [3], which was suggested by Sunada. Suppose for a contradiction that $p$ is not surjective, then $H_{1}(\mathcal{G}, \mathbb{Z}) / p(\mathcal{I}(\sigma))$ is non-trivial. Hence we can find a finite factor $G$ and, by construction, $p_{G}: \mathcal{I}(\sigma) \rightarrow G$ is the trivial map. This contradicts Proposition 1.

Let $\mathcal{I}_{0}(\sigma) \subset \mathcal{I}(\sigma)$ correspond to those elements in $\mathcal{I}$ which are null in homology.

COROLlary 2. We have that $H_{1}(\mathcal{G}, \mathbb{Z}) \cong \mathcal{I}(\sigma) / \mathcal{I}_{0}(\sigma)$.

Proof. This follows immediately since $I_{0}(\sigma)=$ ker $p$.

Fix a skew product $\sigma_{f}$ and a finite abelian group $G$ and let $\pi: \widetilde{\mathcal{G}} \rightarrow \mathcal{G}$ be the covering map. This induces a map $\pi_{*}: H_{1}(\widetilde{\mathcal{G}}, \mathbb{Z}) \rightarrow H_{1}(\mathcal{G}, \mathbb{Z})$ between homology groups, whose image we denote by $\pi_{*}\left(H_{1}(\widetilde{\mathcal{G}}, \mathbb{Z})\right) \subset H_{1}(\mathcal{G}, \mathbb{Z})$.

A closed orbit $\tilde{\tau}$ for $\sigma_{f}$ projects to a closed orbit $\tau$ for $\sigma$ repeated $n(\tau)$ times, say. We associate to a closed orbit $\tau$ its norm, defined by $N(\tau)=\tau^{n(\tau)} \in \mathcal{I}(\sigma)$. This extends to a homomorphism $N: \mathcal{I}\left(\sigma_{f}\right) \rightarrow \mathcal{I}(\sigma)$, whose image we denote by $N\left(\mathcal{I}\left(\sigma_{f}\right)\right) \subset \mathcal{I}(\sigma)$. Observe that $N\left(\mathcal{I}\left(\sigma_{f}\right)\right)$ is contained in the kernel of the Frobenius map $p_{G}: \mathcal{I}(\sigma) \rightarrow G$.

We will write $p^{f}: \mathcal{I}\left(\sigma_{f}\right) \rightarrow H_{1}(\widetilde{\mathcal{G}}, \mathbb{Z})$ for the natural homomorphism. Since $\sigma_{f}$ is assumed to be a mixing subshift of finite type, Corollary 1 shows that $p^{f}$ is surjective. 
THEOREM 1. Let $\sigma_{f}$ be a Galois extension of $\sigma$ by a finite group $G$. Then there is a surjective homomorphism

$$
\phi: G \rightarrow \frac{\mathcal{I}(\sigma)}{\mathcal{I}_{0}(\sigma) N\left(\mathcal{I}\left(\sigma_{f}\right)\right)},
$$

and $\phi$ is an isomorphism if and only if $G$ is abelian. In particular, $\mathcal{I}_{0}(\sigma) N\left(\mathcal{I}\left(\sigma_{f}\right)\right)<\mathcal{I}(\sigma)$ is a subgroup of index at most $|G|$, with equality if and only if $G$ is abelian. Furthermore, if $G$ is abelian then the map $\tau \rightarrow[\tau]_{G}$ induces an isomorphism

$$
\frac{\mathcal{I}(\sigma)}{\mathcal{I}_{0}(\sigma) N\left(\mathcal{I}\left(\sigma_{f}\right)\right)} \rightarrow G
$$

Proof. Our proof is inspired by the approach in [5]. By construction the following diagram commutes, where $p$ and $p^{f}$ are surjective.

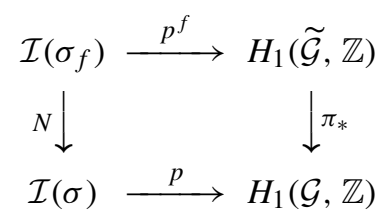

Hence, we have that

$$
\frac{\mathcal{I}(\sigma)}{\mathcal{I}_{0}(\sigma) N\left(\mathcal{I}\left(\sigma_{f}\right)\right)} \cong \frac{H_{1}(\mathcal{G}, \mathbb{Z})}{\pi_{*}\left(H_{1}(\widetilde{\mathcal{G}}, \mathbb{Z})\right)} .
$$

Using the Hurewicz maps $h: \pi_{1}(\mathcal{G}) \rightarrow H_{1}(\mathcal{G}, \mathbb{Z})$ and $\tilde{h}: \pi_{1}(\widetilde{\mathcal{G}}) \rightarrow H_{1}(\widetilde{\mathcal{G}}, \mathbb{Z})$ we have the following commuting short exact sequences.

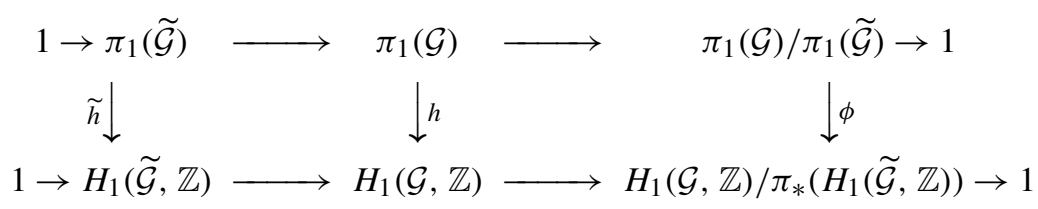

Since the Hurewicz maps are surjective, the final induced homomorphism

$$
\phi: \pi_{1}(\mathcal{G}) / \pi_{1}(\widetilde{\mathcal{G}}) \rightarrow \frac{H_{1}(\mathcal{G}, \mathbb{Z})}{\pi_{*}\left(H_{1}(\widetilde{\mathcal{G}}, \mathbb{Z})\right)} \cong \frac{\mathcal{I}(\sigma)}{\mathcal{I}_{0}(\sigma) N\left(\mathcal{I}\left(\sigma_{f}\right)\right)}
$$

is also surjective. Moreover, since $\widetilde{\mathcal{G}}$ is a Galois cover for $\mathcal{G}$, we can write that $G \cong \pi_{1}(\mathcal{G}) / \pi_{1}(\widetilde{\mathcal{G}})$, giving the required surjective homomorphism $\phi$.

If $\phi$ is an isomorphism then $G$ is clearly abelian. Conversely, if $G \cong \pi_{1}(\mathcal{G}) / \pi_{1}(\widetilde{\mathcal{G}})$ is abelian then there is a natural homomorphism $\psi: H_{1}(\mathcal{G}, \mathbb{Z}) \rightarrow G$, since $\widetilde{\mathcal{G}}$ is a quotient of the universal homology cover of $\mathcal{G}$, for which the covering group is $H_{1}(\mathcal{G}, \mathbb{Z})$. We will show that $\operatorname{ker}(\phi)$ is trivial. Assume that $g \in \operatorname{ker}(\phi)$ then we can choose $\beta \in H_{1}(\mathcal{G}, \mathbb{Z})$ such that $\psi(\beta)=g$. Since $\beta$ is in the kernel of the homomorphism $H_{1}(\mathcal{G}, \mathbb{Z}) \rightarrow H_{1}(\mathcal{G}, \mathbb{Z}) /$ $\pi_{*}\left(H_{1}(\widetilde{\mathcal{G}}, \mathbb{Z})\right)$ we have that $\beta \in \pi_{*}\left(H_{1}(\widetilde{\mathcal{G}}, \mathbb{Z})\right)$. In particular, we can choose $\alpha \in H_{1}(\widetilde{\mathcal{G}}, \mathbb{Z})$ such that $\pi_{*}(\alpha)=\beta$. Finally, we can choose $\gamma \in \pi_{1}(\widetilde{\mathcal{G}})$ which projects to $1 \in \pi_{1}(\mathcal{G}) / \pi_{1}(\widetilde{\mathcal{G}})$ because of the short exact sequence property. Since the diagram commutes we deduce that $g=1$, as required.

To prove the final claim in the theorem, we can define a homomorphism $\psi: I(\sigma) / I_{0}(\sigma) N\left(I^{f}\right) \rightarrow G$ in the following way. Given any coset in $I(\sigma) / I_{0}(\sigma) N\left(I^{f}\right)$ 
we can choose a representative element in $\mathcal{I}(\sigma)$ and then apply the map $p_{G}$ to it. In order to see that this is well defined, we observe that $\mathcal{I}_{0}$ and $N\left(\mathcal{I}\left(\sigma_{f}\right)\right)$ are both contained in $\operatorname{ker}\left(p_{G}\right)$, and hence so is $\mathcal{I}_{0} N\left(\mathcal{I}\left(\sigma_{f}\right)\right)$. However, since $I(\sigma) / I_{0}(\sigma) N\left(I^{f}\right)$ and $G$ have the same cardinality, we deduce that $\psi$ is an isomorphism.

It would be interesting to find a number theoretical result which can be recovered using this dynamical result.

\section{Anosov flows}

Theorem 1 has natural analogue for transitive Anosov flows. In this section we briefly describe these results.

Let $T_{t}: M \rightarrow M$ be a transitive Anosov flow. (We do not assume that $T_{t}$ is mixing.) As before, we let $\tau$ denote a typical closed orbit of the flow and $\mathcal{I}(T)$ the free abelian group generated by all $\tau$. For a finite group $G$, let $\widetilde{M}$ be a Galois $G$-cover of $M$ and let $\widetilde{T}_{t}: \widetilde{M} \rightarrow \widetilde{M}$ be the lifted flow. Clearly, $\widetilde{T}_{t}$ is again an Anosov flow.

For every closed $T$-orbit $\tau$, we can associate a Frobenius class $[\tau]_{G} \subset G$ (cf. [3]). If $G$ is abelian then $[\tau]_{G}$ is a well-defined element and the correspondence extends to a homomorphism $p_{G}: \mathcal{I}(T) \rightarrow G$. In addition, the map sending a closed orbit $\tau$ to its homology class $[\tau] \in H_{1}(M, \mathbb{Z})$ induces a natural homomorphism from $p: \mathcal{I}(T) \rightarrow$ $H_{1}(M, \mathbb{Z})$. By the Chebotarev theorem [3], every conjugacy class in $G$ is represented by a closed orbit. In particular, for each finite $G, p_{G}$ is a surjection and hence, as in $\S 1, p$ is a surjection.

Let $\mathcal{I}_{0}(T) \subset \mathcal{I}(T)$ be the elements null in homology, i.e. $\mathcal{I}_{0}(T)=\operatorname{ker} p$. Then $H_{1}(M, \mathbb{Z}) \cong \mathcal{I}(T) / \mathcal{I}_{0}(T)$. A closed $\widetilde{T}$-orbit $\widetilde{\tau}$ on $\widetilde{M}$ projects to a closed $T$-orbit $\tau$ on $M$ repeated $n(\tau)$ times, say. We associate to $\tilde{\tau}$ its norm $N(\tilde{\tau})=\tau^{n(\tau)} \in \mathcal{I}(\rho)$. This extends, in the obvious way, to a homomorphism $N: \mathcal{I}(\tilde{T}) \rightarrow \mathcal{I}(T)$, whose image we denote by $N(\mathcal{I}(\widetilde{T}))$.

The following is an analogue of the Artin reciprocity theorem for Anosov flows. The proof is identical to that for Theorem 1 , with the covering theory of the manifold $M$ replacing that of the graph $\mathcal{G}$.

THEOREM 2. Let $T_{t}: M \rightarrow M$ be a transitive Anosov flow. For a finite group $G$, let $\widetilde{M}$ be a Galois $G$-cover of $M$ and let $\widetilde{T}_{t}: \widetilde{M} \rightarrow \widetilde{M}$ be the lifted flow. Then there is a surjective homomorphism

$$
\phi: G \rightarrow \frac{\mathcal{I}(T)}{\mathcal{I}_{0}(T) N(\mathcal{I}(\widetilde{T}))},
$$

and $\phi$ is an isomorphism if and only if $G$ is abelian. In particular, $\mathcal{I}_{0}(T) N(\mathcal{I}(\widetilde{T}))<\mathcal{I}(T)$ is a subgroup of index at most $|G|$, with equality if and only if $G$ is abelian. Furthermore, if $G$ is abelian then the map $\tau \rightarrow[\tau]_{G}$ induces an isomorphism

$$
\frac{\mathcal{I}(T)}{\mathcal{I}_{0}(T) N(\mathcal{I}(\widetilde{T}))} \rightarrow G .
$$

Remark. This was proved for geodesic flows by Sunada [5]. 
Acknowledgement. The first author if grateful to Professor Sunada for making available a copy of his unpublished article [5].

\section{REFERENCES}

[1] J. W. S. Cassels and A. Frolich. Algebraic Number Theory. Academic Press, New York, 1967.

[2] H. Lenstra and P. Stevenhagen. Artin reciprocity and Mersenne primes. Nieuw Arch. Wiskd. (5) 1 (2000), 44-54.

[3] W. Parry and M. Pollicott. The Chebotarov theorem for Galois coverings of Axiom A flows. Ergod. Th. \& Dynam. Sys. 6 (1986), 133-148.

[4] W. Parry and M. Pollicott. An analogue of Bauer's theorem for closed orbits of skew products. Ergod. Th. \& Dynam. Sys. 28 (2008), 535-546.

[5] T. Sunada. Tchebotarev's density theorem for closed geodesics in a compact locally symmetric space of negative curvature. Preprint.

\section{CAMBRIDGE JUURALS}

